\title{
A Resettable In-Line Particle Concentrator Using AC Electrokinetics for Distributed Monitoring of Microalgae in Source Waters
}

\author{
Quan Yuan ${ }^{1}$, Jayne $\mathrm{Wu}^{1, *}$, Elias Greenbaum², Barbara R Evans ${ }^{2}$ \\ 1. Dept. of Electrical Engineering and Computer Science, the University of Tennessee, Knoxville, TN \\ 37996, USA \\ 2. Chemical Sciences Division, Oak Ridge National Laboratory*, Oak Ridge, TN 37831, USA
}

\begin{abstract}
:
Green algae have been studied as an important and effective biomarker to indicate water quality due to their sensitivity to toxic agents in freshwater sources. However, conventional methods to monitor algal physiology use a chlorophyll fluorometer whose use is hampered by high-cost, large footprint, and limited sensitivity for practical samples containing low algal concentration. To overcome these constraints, we developed a multi-level electrode platform for resettable trapping of algae via AC electro-osmosis (ACEO) and negative dielectrophoresis. Preliminary experiments were performed in freshwater with conductivity of $0.02 \mathrm{~S} / \mathrm{m}$. Algal trapping was demonstrated at a low voltage of $2 \mathrm{~V}$. The concentration effect was experimentally verified by measuring the fluorescence intensity of algae and using hemocytometer counting chambers at the inlet and outlet of the multilevel microchannel lab-on-a-chip. An optimal frequency was found for trapping, which agrees with the frequency dependence of ACEO flow velocity. Through-flow rate and electrode dimensions were optimized as well. Trapping efficiencies within the range of $26 \%-65 \%$ have been obtained. A maximum trapping rate of 182 cells/s was obtained with a flow rate of $20 \mu \mathrm{l} / \mathrm{min}$. This lab-on-a-chip shows high potential for improving the limit of detection in algal monitoring and enabling the development of a portable, integrated and automated system for monitoring the quality of source drinking waters.
\end{abstract}

Key words: Microfluidics; AC electrokinetics; in line particle trapping; microalgae; biosensors; source water monitoring 


\section{Introduction}

Green microalgae are very sensitive to many toxic agents including most pesticides and herbicides. In the presence of these toxic compounds, microalgal autofluorescence changes. Due to this property, algae have been used as a convenient and useful bio-marker for continuous real-time monitoring of freshwater quality in aquatic ecosystems [1]. However, naturally occurring concentration levels of microalgae are typically very low, less than 1.5 $\mu \mathrm{g} / \mathrm{mL}$ in source water. This poses a challenge to real-time monitoring of toxins using algal fluorescence [1-4]. Currently, there are a limited number of instruments available for the detection of algal fluorescence, but they generally require complicated, expensive optical components, which are often bulky and too power intensive to be readily portable [5-11]. None offers simultaneously high sensitivity, field use, and real-time response for distributed algae monitoring. In order to solve this problem, we developed an in-line and resettable particle concentration (cell trapping) mechanism on a chip for sensitive, real-time, long-term, and low cost monitoring of toxin levels in source waters.

A variety of particle concentration methods exist, including optical tweezers [12-14], hydrodynamic trapping [15-18], magnetic trapping [19-23], acoustic trapping [24-25] and dielectrophoresis (DEP) [26-28]. However, most existing methods are unable to achieve simultaneously rapidity, high throughput, repeated use, and low cost. The optical tweezer offers precise control with high resolution for a single cell, but it is of low throughput. Also, it may cause extensive sample heating and thus damage to bio-particles (cell, protein, etc.) [14]. In addition, most optical trapping methods require expensive, sophisticated equipment 
with high power consumption, making it unfeasible for distributed real-time monitoring. Magnetic trapping can perform effective trapping for diamagnetic microparticles. While it is low cost and relative easy to perform, this technique is an offline method requiring lengthy incubation to label target bioparticles with ferromagnetic beads [23]. Another method, hydrodynamic trapping by microstructures, is capable of achieving rapid cell entrapment with a relatively simple structure and large throughput. However, in this method contact of bioparticles with a surface is unavoidable which could lead to irreversible attachment and make the device non-reusable. Acoustic wave has also been used for trapping bioparticles or microparticles in noncontact mode. Acoustic trapping can yield relatively high trapping efficiency for particles larger than $10 \mu \mathrm{m}$ in size. However, its trapping effectiveness gets attenuated drastically for particles with smaller sizes $(<3 \mu \mathrm{m})$. As acoustic traps typically require sheath flows controlled by multiple pumps and a high frequency power supply for generation of acoustic wave generation, it is rather difficult to make the device in a portable format [24-25]. Dielectrophoresis(DEP) has also been used for trapping. As an electrical method, it has the potential to realize a low cost, integrated, rapid and high throughput trapping device for simultaneous concentration of multiple cells, provided that the (di)electric properties of the particle are different from that of the medium. Depending on the permittivities and conductivities of the particles and the medium at the frequency of operation, the particle can be either attracted toward the higher electrical field (positive DEP, i.e. pDEP) or pushed away from it (negative DEP, i.e. nDEP). pDEP could cause the particles to adhere permanently to electrode surfaces, which makes the trapping process irreversible, while 
$\mathrm{nDEP}$ could be implemented for repeated algae concentration, such as direct current (DC) insulator-based dielectrophoresis (iDEP). iDEP uses insulated posts in a DC electric field to generate a non-uniform field and thus trap bio-particles around the posts. Using iDEP, several different research groups demonstrated trapping of bioparticles such as Escherchia-coli bacteria, breast cancer cells, and Bacillus cereus bacteria [29-31]. However, i-DEP trapping requires extremely high DC voltage with field strength above $60,000 \mathrm{~V} / \mathrm{m}$. It is not practical to use such a high voltage in an integrated microfluidic system for distributed monitoring applications.

To address all these limitations for chip-based bioparticle enrichment, we developed a resettable in-line microfluidic platform based on AC electroosmotic (ACEO) effects generated by a 3 dimensional (3D) electrode multichannel system. A parallel plate electrode configuration using a top electrode and bottom mesh electrode, is adopted that, under an appropriate AC signal, induces ACEO flow around the mesh electrode to extract bioparticles from through-flow for enrichment. The same AC signal also induces $\mathrm{nDEP}$ to avoid bioparticle adhesion to the electrodes. In this work, as the first step, the dielectropheretic property of model microalgae in water was characterized to evaluate the feasibility of trapping algae by the combined effects of AC electroosmosis (ACEO) and nDEP. Therefore, a frequency range that is suitable for inducing both ACEO and nDEP was identified. Next, bioparticle concentration experiments were conducted. Both fluorescence light intensity and cell counting measurement were used to quantify the concentration effect. Further, trapping efficiency and throughput were optimized with respect to AC frequency, through-flow 
velocity and electrode size. Since a low voltage and low frequency AC signal is used in this system, field deployable, real-time, and distributed monitoring of microalgal physiology in freshwater ecosystems can be achieved. Another advantage of the system is that it can be prototyped quickly and at low cost without using cleanroom facilities, with the total cost less than $\$ 10$ per unit.

\section{Mechanism and Theory}

2.1 Device Structure

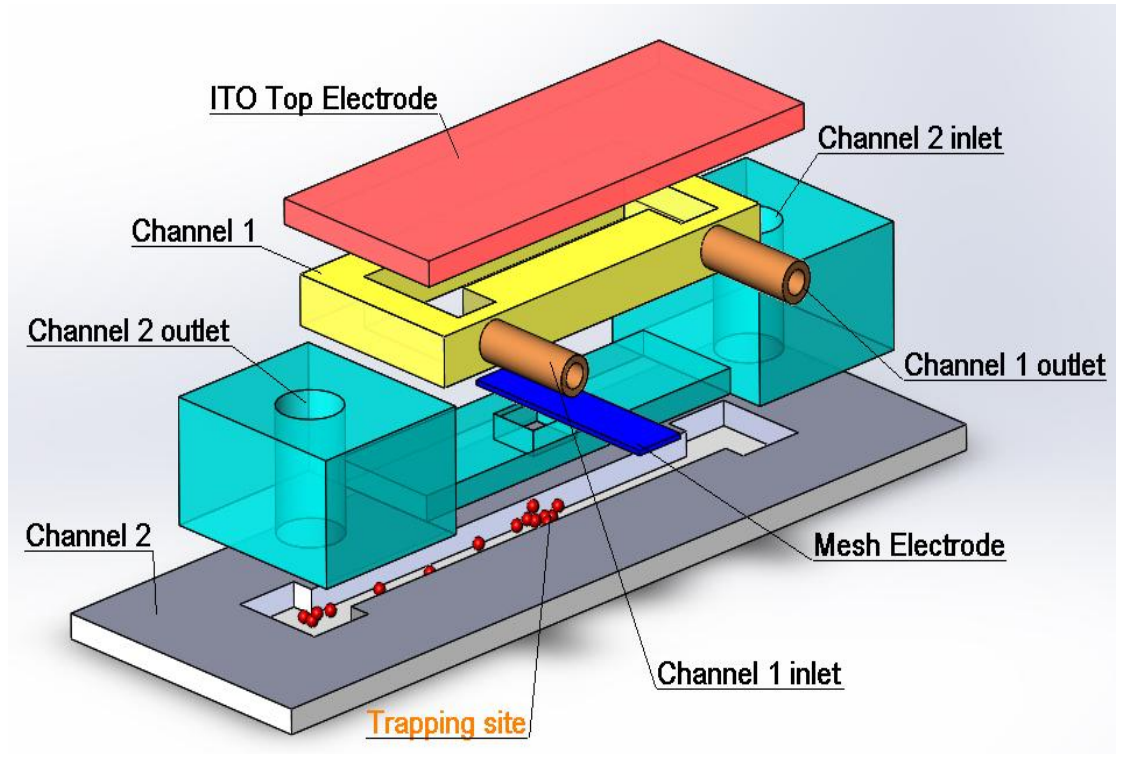

Fig. 1. Schematic illustration of the multilevel electrode lab-chip for resettable particle trapping by combined ACEO and nDEP effects.

The device structure is shown schematically in Fig. 1. It consists of four components.

(1) Supply Channel 1, used to flow the particulate fluid through the electric trapping site, (2)

Channel 2 for particle trapping and release, into which the particles are collected and later on

flushed away by a wash flow. (3) A metallic mesh electrode between Channel 1 and Channel 
2, which functions as a trapping electrode and also allows the passage of particles from Channel 1 to Channel 2. This electrode is made by cutting a strip from a metal filter (06457-AB) manufactured by SPECTRUMLABS. The mesh electrode was woven from 20 $\mu \mathrm{m}$ diameter wires with openings of $45 \mu \mathrm{m} \times 45 \mu \mathrm{m}$. (4) A counter electrode embedded in the top of Channel 1 faces the mesh electrode. An indium-tin-oxide (ITO) slide was used as the counter electrode, which also acted as the top cover and observation window of Channel 1.

An AC signal applied between the mesh and ITO electrodes will induce ACEO effects on the through-flow in Channel 1 and embedded bioparticles. Two types of AC electrokinetic (ACEK) mechanisms worked together to achieve resettable particle trapping in Channel 2. ACEO generates flow motion above and around the mesh electrode, which acted as the major factor to bring particles from Channel 1 through the mesh electrode into Channel 2. nDEP repelled particles from the electrode, to ensure contact-free trapping. Using these two combined mechanisms, contact-free particle trapping was achieved.

\subsection{AC electro-osmosis}

AC electro-osmosis (ACEO) refers to induced flow at the surface of electrodes under the influence of an inhomogeneous AC electrical field. When an electrode is energized by an electrical potential, in order to maintain charge neutrality, counter-ions (ions of polarity opposite to the excitation potential) will be electrostatically attracted to the electrode. Together, the induced counter-ion layer and the electrical surface charge form the so-called electrical double layer, modelled as a capacitor electrically. The induced counter-ions can move under an electric field that is tangential to the electrode surface. Due to the viscosity 
between the ions and fluid, fluid will be dragged by ions to cause fluid movement, hence ACEO flow is formed. A general equation for ACEO fluid velocity is given as [32]; $u_{A C E O}=-\left(\frac{\varepsilon_{m}}{\eta}\right) \cdot \Delta \xi \cdot E$

where $\eta$ and $\varepsilon_{m}$ are the viscosity and permittivity of the medium, $E$ is the tangential electric field and $\Delta \xi$ is the electrical potential across the charged double layer. In this equation, the permittivity and viscosity of the medium can be considered as constant. ACEO velocity has a quadratic dependence on the applied electrical field.

The double layer is like a capacitor and will be charged by $\mathrm{AC}$ electric current through the resistive fluid bulk. If the applied frequency is very high, there will not be enough time to charge the double layer completely, hence the ACEO velocity has a bell shape dependence on frequency. The time averaged expression for ACEO velocity as a function of frequency has been shown to be [33],

$$
u_{A C E O} \propto \frac{\varepsilon_{m} V^{2}}{\eta(1+\delta) L\left[\frac{\omega}{\omega_{c}}+\frac{\omega_{c}}{\omega}\right]}
$$

where $V$ is the applied voltage, $L$ is the electrode spacing (roughly center to center); $\delta$ is an empirical constant; $\omega_{c} \propto(1+\delta) L$ is the peak frequency for ACEO velocity, which is a constant if the medium is not changed. It can be seen from Eq. (2) that around $\omega=\omega_{c}$, ACEO velocity reaches its maximum value. There is ample experimental evidence that ACEO flow velocity depends on applied frequency and fluid conductivity [34].

Typically, a higher fluid conductivity makes the double layer thinner. Therefore, ACEO effects will diminish with conductivity from $10^{-2}$ and $10^{-1} \mathrm{~S} / \mathrm{m}$ and drop to zero 
eventually [35]. ACEO usually demonstrates effective pumping in electrolytes with low conductivities $\left(<10^{-2} \mathrm{~S} / \mathrm{m}\right)$ at low frequency $(<100 \mathrm{kHz})$, inclusive of source waters. The frequency of the applied AC signal will be optimized and presented in the section 4.2. ACEO has been used in multiple microfluidic applications, such as pumping, mixing and particle trapping [34-45]. Mainly two types of electrode designs have been reported for inducing ACEO flows, planar interdigitated microelectrode arrays and parallel plate electrodes with asymmetric dimensions [37-38]. Planar interdigitated electrodes are not compatible with the multichannel and 3D electrode design, so parallel plate electrode design is used here.

Since we are dealing with low voltage and low conductivities, temperature rise of the sample can be neglected. Therefore, the AC electro-thermal (ACET) effect, which is an important method for particle manipulation and bio-fluidic pumping in high conductivity media [46 -47] and is dependent on a temperature gradient, can be neglected.

\subsection{Dielectrophroesis}

DEP is the electrical force exerted on a dielectric particle when it is in a non-uniform electric field. The particles do not need to be charged. Instead, charges will be induced at the particle surface by the electrical field. The strength of DEP force depends on the following factors: particle size, (di)electrical properties (permittivity and conductivity) of the medium and particle, electric field gradient and AC frequency. The DEP velocity can be expressed as [32]

$$
u_{D E P}=\frac{a^{2} \varepsilon_{m}}{6 \eta} \operatorname{Re}\left[f_{C M}\right] \nabla|E|^{2},
$$


where $a$ is the particle diameter, $\eta$ is the fluid viscosity $f_{C M}$ is Clausius-Mossotti factor which is frequency dependent with a value between -0.5 and 1 . The particle is attracted towards the higher field when the CM factor is $>0$, known as positive DEP (pDEP); or pushed away from it when the CM factor is $<0$, known as negative DEP (nDEP). The experiments for identifying the frequency range for $n / p$ DEP are discussed in section 4.1.1. For a microalgal cell, tap water medium was used, which closely matches the ionic strength of fresh water environments that microalgae typically live in. nDEP of microalgae has been observed at frequencies lower than $10 \mathrm{kHz}$. Obviously, the algae velocity experience from DEP force is size dependent. Assume $\operatorname{Re}[\mathrm{fcm}]=-0.5(\mathrm{nDEP})$, viscosity of water is $1.08 \cdot 10^{-3}$ $\mathrm{Pa}$, permittivity of water is $80 \cdot 8.85 \cdot 10^{-12} \mathrm{~F} / \mathrm{m}$. Numerical simulation shows that nDEP will be around $285 \mu \mathrm{m} / \mathrm{s}$ for algae with a $5 \mu \mathrm{m}$ diameter, and $\mathrm{nDEP}$ is $102 \mu \mathrm{m} / \mathrm{s}$ for algae with a $3 \mu \mathrm{m}$ diameter. For any particle less than $1 \mu \mathrm{m}$ in diameter, nDEP is below $2 \mu \mathrm{m} / \mathrm{s}$, which in our case is insignificant when compared to other velocities such as sedimentation velocity.

Other forces such as gravity also have effect on algae trapping. The magnitude of the sedimentation velocity of particles due to gravity can be expressed as $u_{g}=0.2 a^{2}\left(\rho_{p}-\rho_{m}\right) g / \eta$, where $\rho_{\mathrm{m}}$ and $\rho_{\mathrm{p}}$ are the mass density of medium and particle respectively, $\mathrm{g}$ is the gravitational constant. Assume mass density of algae is twice that of medium, the sedimentation velocity of algae is about $15 \mu \mathrm{m} / \mathrm{s}$, which is not predominant but plays a role in algae trapping. 


\section{Experiments}

3.1 Device operation

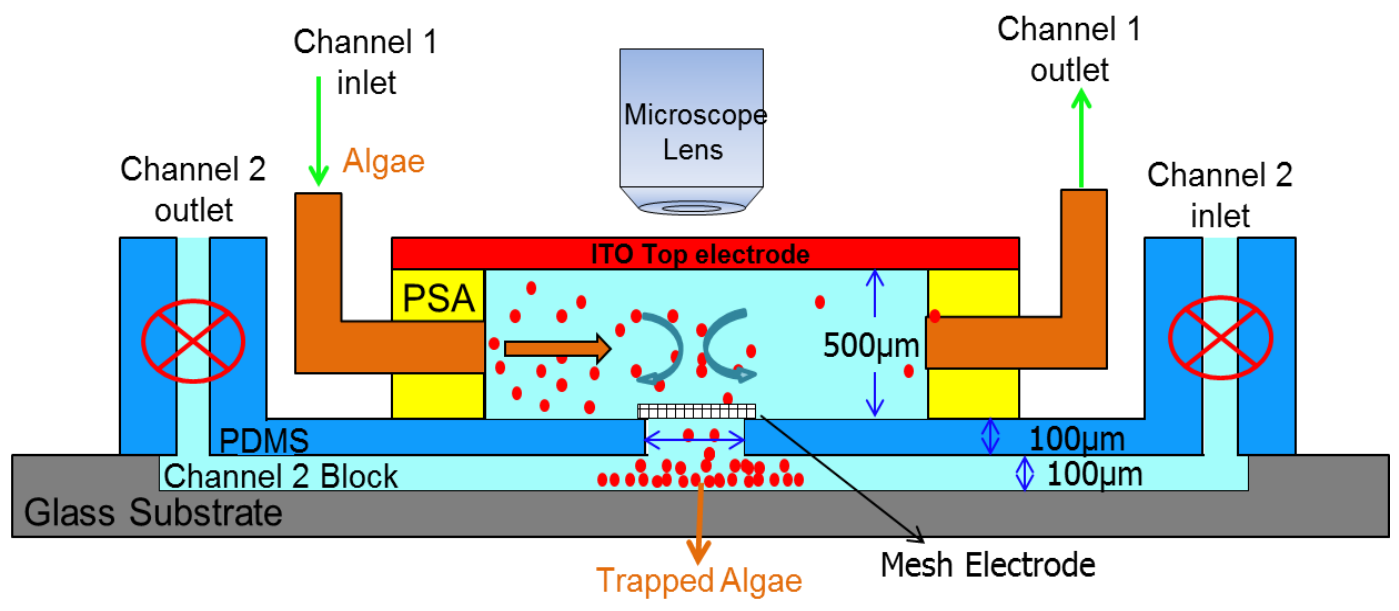

(a)

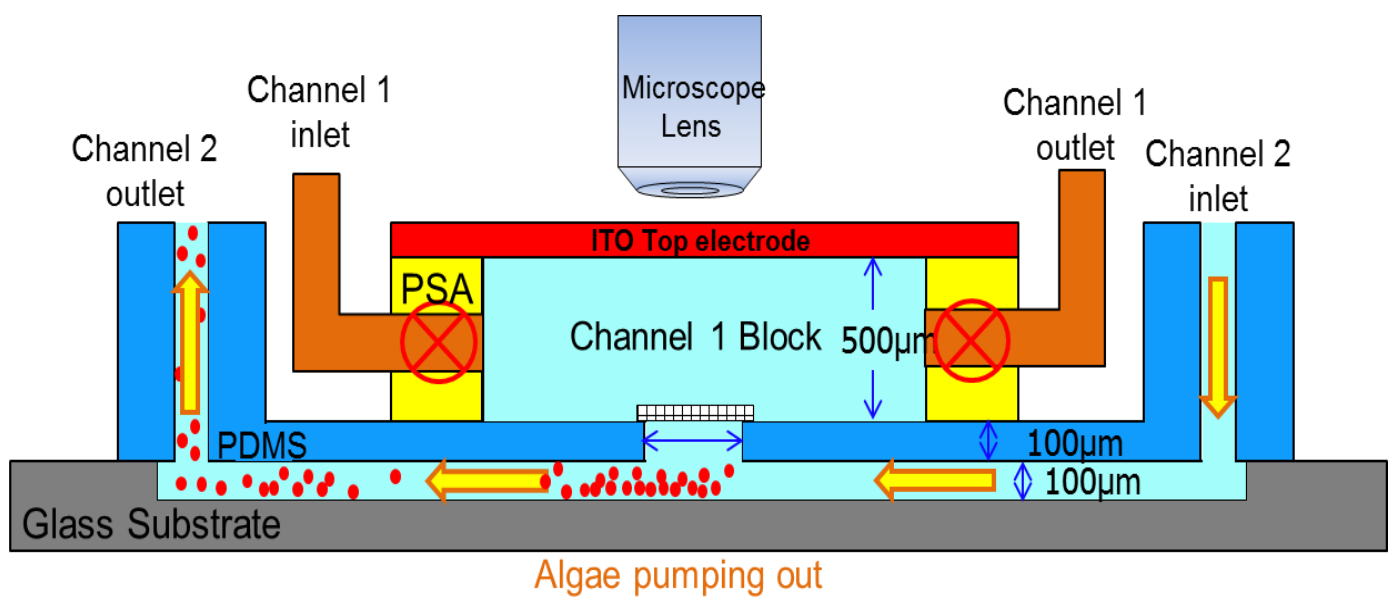

(b)

Fig. 2. Side view of the device during two stages of operation (a) Stage 1: Particle extraction from Channel 1 and concentration in Channel 2, and (b) Stage 2: Particle release/elution from the trapping site in Channel 2 for next cycle of use

The device has two stages of operation in one cycle of "catch and release." The first is the trapping stage and the second is the flushing stage which resets the device for the next test. The trapping and flushing stages are conceptually shown in Fig. 2 (a) and (b). During the trapping stage, Channel 1 is open for sample flow and Channel 2 is shut off. An AC voltage is applied between the top ITO and bottom mesh electrodes across Channel 1. As a result, 
ACEO force and DEP force are induced between the ITO and mesh electrodes, which can exert forces on particles transverse to the flow streamlines and direct them towards the mesh. ACEO flow directions always follow those of tangential electric fields at the electrode surface. In this case, the mesh electrode generates ACEO flow patterns similar to that by an array of orthogonal electrodes as discussed in Yang's work [48]. Here, each mesh wire acts similar to the pin electrode in Yang's work, causing flows going along the wire surface downwards away from the top electrodes. As a result, vortices generated by ACEO flow bring algae particles from a large part of Channel 1 to the mesh electrode area. In the vicinity of mesh electrodes, ACEO, nDEP, and gravity together affect the trajectory of particle movement. nDEP mostly keeps algae from attaching to the electrodes. For the top $1 / 3$ of a wire electrode, and within a very limited distance from an electrode, DEP effect levitates algae particles. However, ACEO flow is stronger, and due to the short range of DEP effect, most of algae particles will follow ACEO flows down to the center of mesh openings. There the direction of nDEP force turns downward. Then the combined effect of gravity, ACEO and nDEP helps to trap algae in Channel 2. Numerical simulation of ACEK velocities was performed in an effort to explain trapping mechanisms. The simulation results are provided in the Supplementary Material section 4. Once inside Channel 2, local flow significantly weakens due to loss of the ACEO effect. Under the influences of gravity and nDEP still at the mesh, most of the particles will be retained in Channel 2. As Channel 2 is shut off during the "catch" stage, algae (particles) accumulate to a detectable level. During the particle release 
stage, Channel 1 will be off and Channel 2 open, and all the collected particles in Channel 2 will be flushed away. Then the next cycle of operation can start.

\subsection{Device fabrication}

Desktop fabrication was used to prototype this bioparticle concentrator. It only takes 4 hour (include 3 hour PDMS curing time) to fabricate one device and the total cost of the device is around \$6.7. One glass slide is used as the base of the device. The channels are made of pressure sensitive adhesive (PSA) tape and cut into desired geometries by a digital craft cutter (Quickutz Silhouette SD). The fabrication procedure is given below:

1. Clean the glass slide with acetone and de-ionized (DI) water and dry with an air gun.

2. Attach 1 layer of PSA tape on the cleaned glass slide uniformly (remove all air bubbles)

3. Cut the attached PSA tape into Channel 2 with openings for inlet and outlet. The dimensions of Channel 2 are $50 \mathrm{~mm} \times 2 \mathrm{~mm} \times 100 \mu \mathrm{m}(1 \times \mathrm{w} \times \mathrm{h})$.

4. Attach two prefabricated PDMS tube holders onto the ends of Channel 2. Embed silicone tubes (ID $1.02 \mathrm{~mm}$, CAT No. 07625-28, Cole-Parmer Instrument Company) into Channel 2 for the flushing step.

5. Place the metal mesh electrode on the top surface of Channel 2.

6. Attach 1 layer of PSA tape as a spacer with the pre-cut opening for the electrode mesh.

7. Place 5 layers of PSA tape together to reach a thickness of $500 \mu \mathrm{m}$ and cut using the craft cutter to form Channel 1. The dimensions of Channel 1 are $15 \mathrm{~mm} \times 2 \mathrm{~mm} \times 500 \mu \mathrm{m}(1 \times \mathrm{w}$ $\times h)$.

8. Attach Channel 1 to the spacer and embed two polyimide coated capillaries (ID $246.6 \mu \mathrm{m}$, 
OD $357.7 \mu \mathrm{m}$, \#106815-0026, Polymicro Technologies) as the inlet and outlet for sample injection.

9. Seal Channel 1 by laying an ITO strip on its top.

Epoxy glue is used to seal any leaks in the device. In order to produce a non-uniform electrical field, the mesh electrode is designed to be $1 \mathrm{~mm}$ wide, forming a trapping area of 1 $\mathrm{mm} \times 2 \mathrm{~mm}$ in Channel 1, which is much smaller than the ITO area. A photograph of the bioparticle concentrator is shown in Fig. 3. The images of the mesh electrode can be found in the Supplementary Material (Fig. S1).

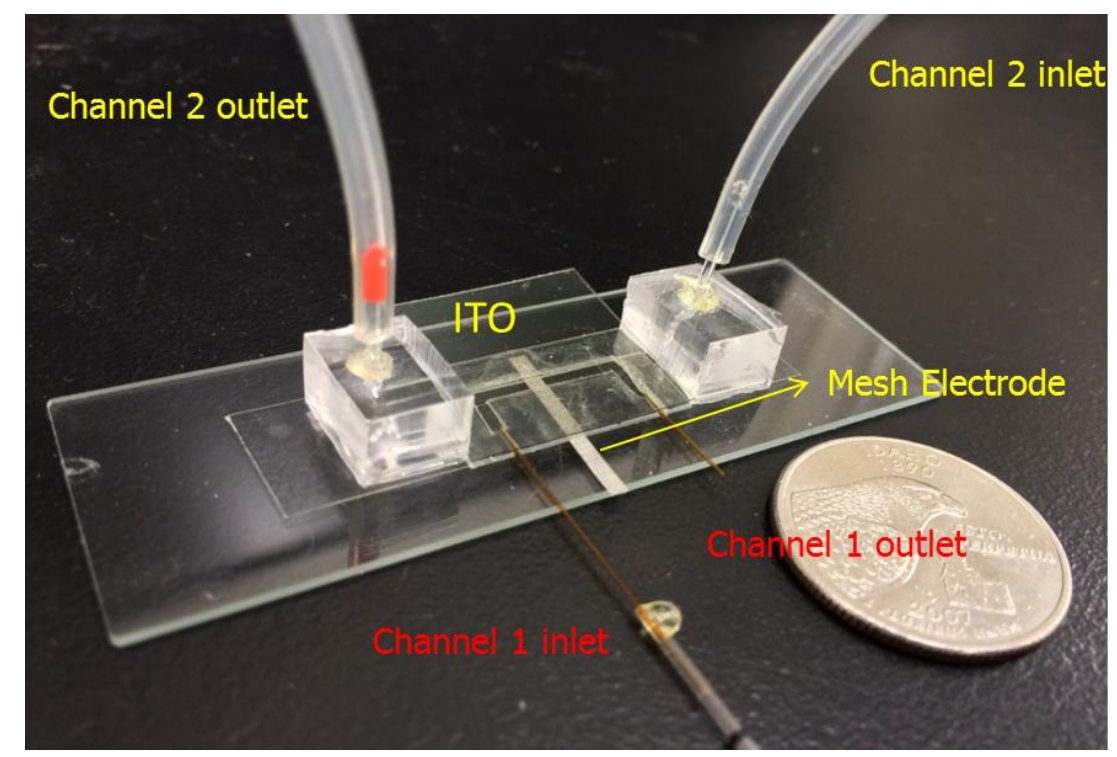

Fig. 3. Photograph showing the resettable in-line ACEK particle concentrator

\subsection{Particle and cell preparation}

The freshwater algal species Chlorella kessleri (UTEX, University of Texas, Austin, Texas, USA) was cultivated in modified Sueoka minimal salts medium [49]. The detailed composition of this medium can be found in the Supplementary Material. The algae are 
grown under static conditions in $50 \mathrm{ml}$ of culture media in $125 \mathrm{ml}$ flasks at $26{ }^{\circ} \mathrm{C}$. Illumination was provided by Sylvania Gro-Lamps in a $12 \mathrm{hr}$ dark/12 hr light diurnal cycle at 1900 lux (33.61 $\mu \mathrm{mol}$ photons $\left.\mathrm{m}^{-2} \mathrm{~s}^{-1}\right)$. The chlorophyll content of the Chlorella algae was determined to be $1.16 \mu \mathrm{g}$ chl/cell by calculation from the chlorophyll $(\mathrm{a}+\mathrm{b})$ concentration determined in triplicate from methanol extracts [50] and cell counts with a Hausser Scientific Bright Line Counting Chamber cell counting chamber. The algae stock solution is then diluted 10 times with freshwater buffer to reduce the concentration to 1266 cells $/ \mu 1$. This diluted sample was used for all the experiments. The electrical conductivity of the algae sample buffer was $0.02 \mathrm{~S} / \mathrm{m}$ (Hanna Instruments HI 98129 conductivity meter). Chlorella cells are spherical, with a diameter of $\sim 5 \mu \mathrm{m}$ when in suspension. Microscopic images of the algae are given in the Supplementary Material (Fig. S2).

\subsection{Experimental setup}

Sampling and flushing flows through the particle concentrator were produced by two external microsyringe pumps (NE-1000 programmable single syringe pump, Pump systems Inc), one for each channel. Channel 1 was primed with sampling fluid with algae suspension for trapping, and Channel 2 was prefilled with purified freshwater buffer. During trapping experiments, the pump for Channel 2 was shut off, and the pump for Channel 1 stayed on to provide the sampling flow with an average linear velocity of $83-416 \mu \mathrm{m} / \mathrm{s}$. The syringe pump for Channel 1 was put on a shaker during injection to keep algae suspended in the fluid throughout the experiment. 
The AC trapping signal was generated by a $50 \mathrm{MHz}$ function/pulse signal generator 8551 (Tabor Electronics Ltd, USA). An oscilloscope MSO6012A (Agilent technology, USA) was used to monitor the actual voltage drop over the electrode in real-time. The applied AC signal on the electrodes was $2 \mathrm{~V}$. The AC frequency was varied to optimize trapping effect.

Due to fluorescence from the chlorophyll in algae, the particle concentration can be represented by the light intensity through a CCD camera. A Nikon eclipse LV100 microscope with CoolSnap photometrics CCD was used to measure the light intensity in gray scale. Image processing software "Image-Pro 3D suite" was used for subsequent data analysis. The CCD measurement area was $500 \mu \mathrm{m} \times 500 \mu \mathrm{m}$, in the central part of the mesh electrode trapping site $(1 \mathrm{~mm} \times 2 \mathrm{~mm})$. The gray scale of a clean device prior to trapping was used as the background control. The average gray scale of the whole measurement area was used to represent the light intensity from the trapped algae. Exposure time was fixed at $125 \mathrm{~ms}$ for all cases to ensure accuracy in measuring light intensity of the trapped algae. Image-Pro 3DS software was also used for particle image velocimetry analysis to characterize micro-flow velocities.

\section{Results and discussion}

\subsection{ACEK Experimental Characterization}

As discussed earlier, both ACEO and nDEP were used for resettable trapping of bioparticles. Both ACEO and DEP effects are frequency dependent. ACEO flows usually occur in the low frequency range, and DEP can change between pDEP (attraction) and nDEP (repulsion) with frequency. In the following, the frequency dependence of ACEO flows 
generated by the mesh electrodes and the n/p DEP of Chlorella in fresh water were characterized.

\subsection{1 n/p DEP of Chlorella}

In order to achieve repeated use of a particle trap, non-contact trapping of algae is necessary. Therefore, it is critical that at the $\mathrm{AC}$ frequency of operation algae will experience $\mathrm{nDEP}$ and will not adhere to the electrodes.

Mesh electrodes embedded within a commercial microchamber (SecureSeal $^{\mathrm{TM}}$ Hybridization Chambers, Grace Bio-lab Inc) were used to conduct the DEP characterization experiment of the algae suspension. Since DEP is dependent on the (di)electric properties of the solution, tap water with a conductivity of $0.02 \mathrm{~S} / \mathrm{m}$ was used in the experiments, which is very close in conductivity to the freshwater that Chlorella naturally lives in. In order to avoid electrochemical reactions, the applied voltage was limited at $2 \mathrm{~V}$ in amplitude. The applied frequency was varied from $1 \mathrm{kHz}$ to $20 \mathrm{MHz}$ in roughly logarithmic increments. The frequency regions of positive and negative dielectrophoreis exhibited by Chlorella algae were observed and recorded.

pDEP of Chlorella was observed when the applied frequency was above $5 \mathrm{MHz}$, at which point ACEO flow was virtually non-existent. After applying the AC signal, Chlorella particles became trapped at the electrode edge, and continuous fluid motion indicative of micro-flows could not be observed. Trapping happens only in the region very closed to the electrode edges. nDEP of Chlorella was observed at frequencies below $10 \mathrm{kHz}$ and coexisted with the ACEO effect. The particles were repelled from the electrodes, and strong fluid 
motion was formed and circulated continuously at the surface of the electrodes. Therefore, the AC frequency for our device was chosen to be below $10 \mathrm{kHz}$.

\subsubsection{ACEO}

It is well established that ACEO flow velocity varies with applied frequency. Therefore, the frequency dependence of ACEO velocity was characterized using electrodes with the same characteristic length as that of the ITO/mesh electrode pair.

A stainless steel wire electrode with a diameter similar to that of the mesh electrode $(20 \mu \mathrm{m})$ was used as the test electrode, and an ITO slide was used as the counter-electrode, to simulate ACEO flow induced by the ITO mesh electrode pair. The $2 \mathrm{~V}$ AC signal from 300 $\mathrm{Hz}$ to $10 \mathrm{kHz}$ was applied to the electrodes. Data in Fig. 4 were obtained by measuring algae velocities at positions with a distance of $5 \mu \mathrm{m}$ to the electrode edge in lateral direction and 5 $\mu \mathrm{m}$ above the electrode top surface. The results show that the flow velocity reaches a maximum at around $500 \mathrm{~Hz}$. Therefore, a $2 \mathrm{~V}$ AC signal at $500 \mathrm{~Hz}$ was used in the subsequent trapping experiments. 


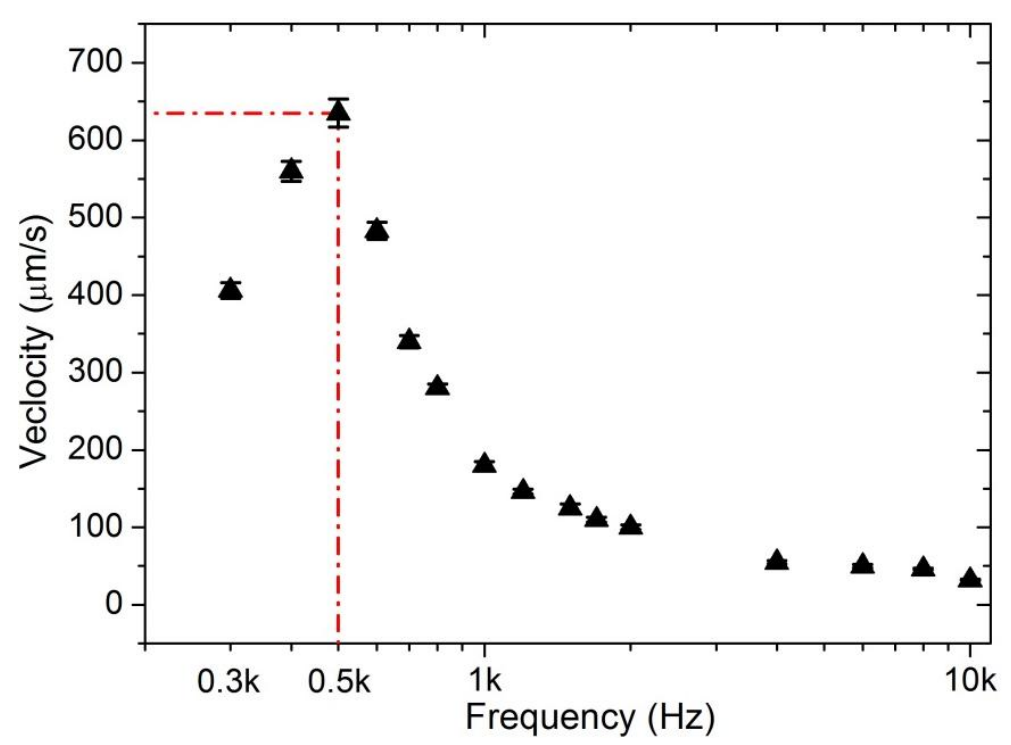

Fig. 4. Measured ACEO velocities as a function of applied frequency.

The optimal ACEO frequency can also be estimated based on the fact that ACEO effect depends on the charging of electrical double layer capacitors through the fluid resistance between two electrodes. The optimal angular frequency can be estimated as $\omega_{\mathrm{c}}=$ $2 \cdot \sigma \cdot \lambda /(\varepsilon \cdot d)$ [32], where $\sigma$ is the conductivity of electrolyte, and $\lambda$ is the Debye length of electrical double layer. $d$ is the distance between two parallel electrodes, which is around 400 $\mu \mathrm{m}$ in our case. The conductivity of our sample medium is $0.02 \mathrm{~S} / \mathrm{m}$, and $\varepsilon=\varepsilon_{0} \varepsilon_{\mathrm{r}}=80 \cdot 8.85 \cdot 10^{-12} \mathrm{~F} / \mathrm{m}$ for aquous solution. The thickness of double layer in river water is around $10 \mathrm{~nm}$. The optimal frequency is found to be $\mathrm{f}_{\mathrm{c}}=\sigma^{\cdot} \lambda /\left(\pi^{\cdot} \varepsilon^{\cdot} d\right)=449.6 \mathrm{~Hz}$, in good agreement with the experiments.

\subsubsection{Proof of Concept for resettable particle Trapping}

Next, Chlorella trapping was demonstrated with a $500 \mathrm{~Hz}$ AC signal at $2 \mathrm{~V}$ amplitude. Before applying voltage, there was no sign of algae in Channel 2 below the mesh electrode, 
except for some floating algae. When a $2 \mathrm{~V} 500 \mathrm{~Hz}$ AC signal was applied, Chlorella trapping started. Trapped algae were retained in Channel 2 under the mesh electrode. With an AC signal continuously applied over the electrodes, the algae started forming clusters and the clusters become larger and brighter as the through-flow continuously brought in more algae. Fig. 5 (a)-(d) demonstrates the time-lapsed trapping effect for a 180 second duration. Based on fluorescence intensity, algae accumulated by a factor of 6 over the original density in 180 seconds. When the voltage was removed, the trapped algae were released rapidly and washed away by an external flush flow through Channel 2, as shown in Fig. 5 (e)-(f). After the washing and cleaning steps, the device was successfully reset and ready for the next trapping cycle.

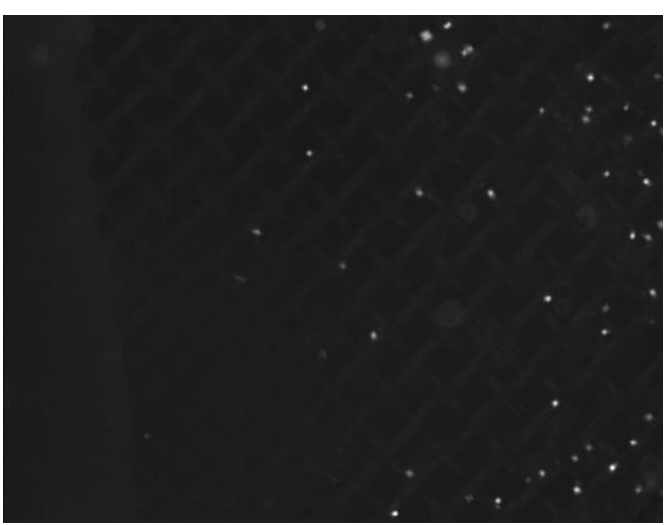

(a)

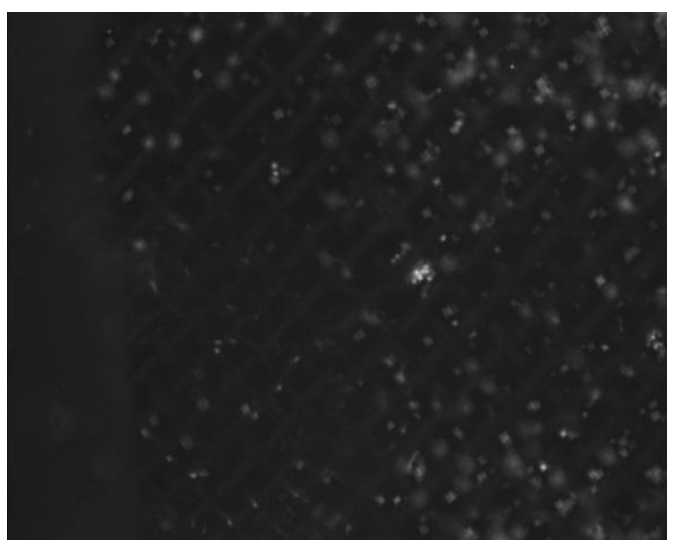

(c)

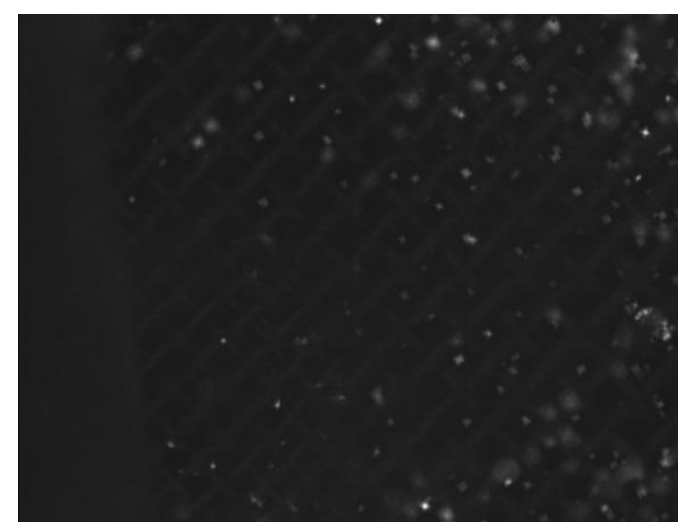

(b)

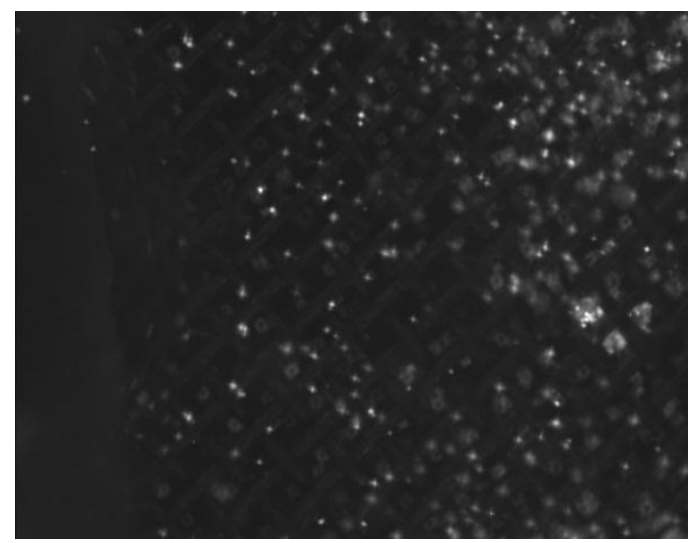

(d) 


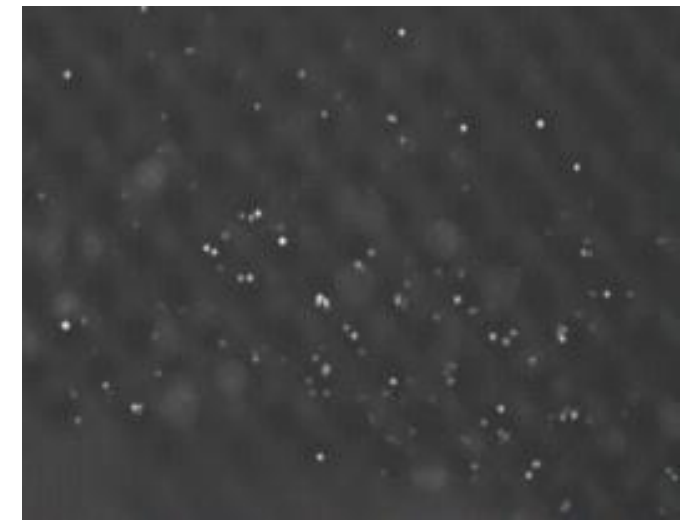

(e)

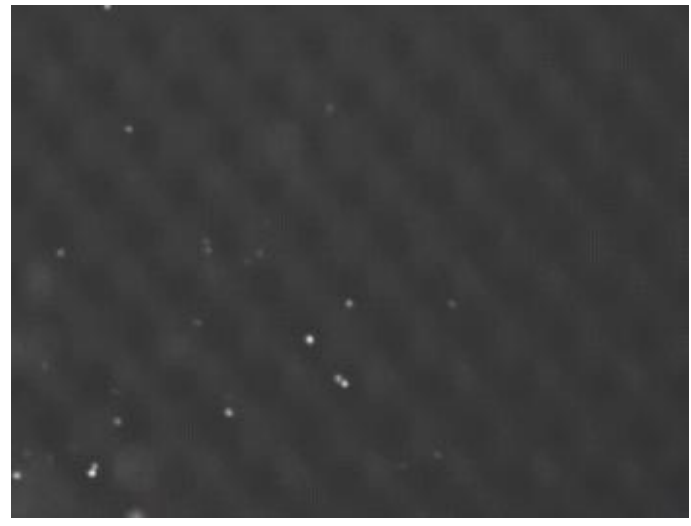

(f)

Fig.5. Still images demonstrating the entire procedure of Chlorella trapping within 180 seconds.(a) Start time, (b) Trapping at 60 seconds, (c) Trapping at 120 seconds, (d) Trapping at 180 seconds, (e) Device reset: before washing, (f) Device reset: after washing. In these images, the focal plane is below the mesh in order to view Chlorella.

To verify the role of the AC electric field in trapping, we also fabricated a particle concentrator chip with two mesh electrodes built between Channels 1 and 2, one mesh electrode as the control mesh with the other as the active trapping electrode. The control mesh is identical in size and material to the trapping mesh electrode, except that there is no electrical potential connected to the control mesh. As the sample fluid was pumped through Channel 1, it flowed over the control mesh and then the trapping mesh. It was observed that Chlorella only accumulated under the trapping mesh electrode but not the control mesh, corroborating the trapping effect by the AC electric field. Details of this experiment can be found in the Supplementary Material.

\subsection{Optimization of particle concentration}

\subsubsection{Optimization of AC frequency}




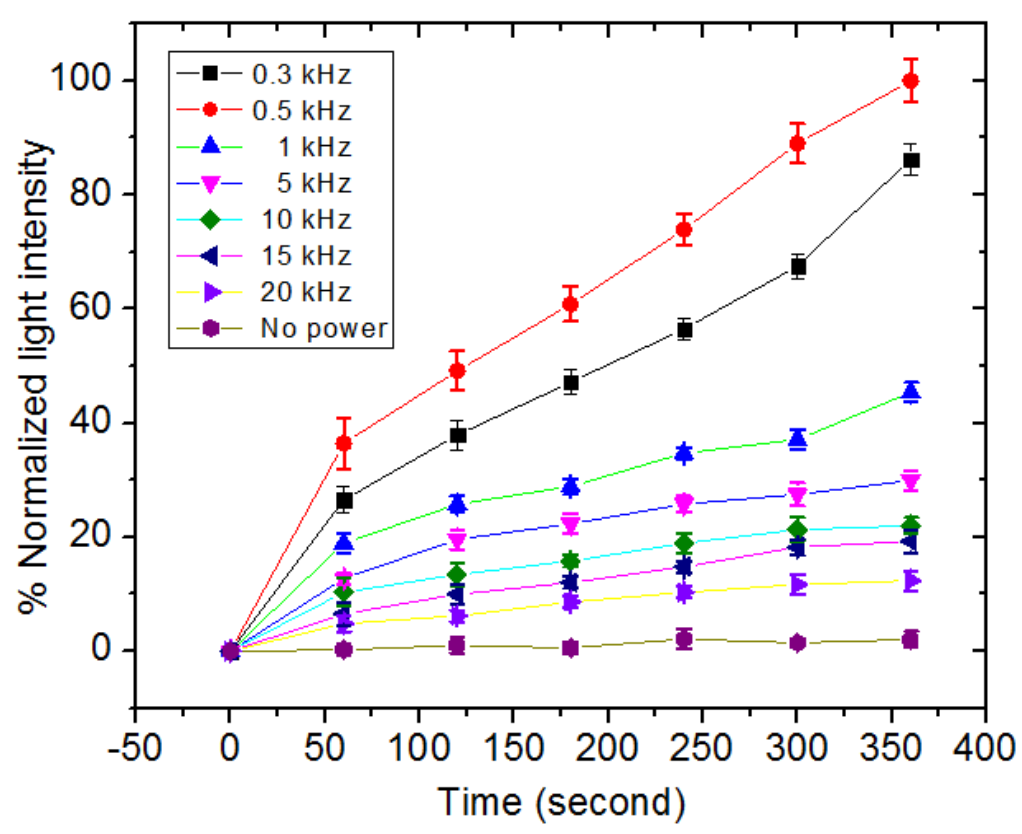

Fig. 6. Normalized light intensity change with time when the particle concentrator operated at different frequencies.

Since the ACEO effect is frequency dependent, the trapping effect was expected to be frequency dependent as well. The trapping effect was quantified by measuring the grey scale of algae trapping images. Fig. 6 shows the light intensity as a function of time when using AC signals of different frequencies. The mesh electrode with $45 \mu \mathrm{m}$ opening was used for this optimization with an applied voltage of $2 \mathrm{~V}$ and the external flow rate was set to 15 $\mu \mathrm{l} / \mathrm{min}$. It is clear from Fig. 6 that the concentration effect demonstrated the same frequency dependence as that of the ACEO velocity. The optimized frequency was around $500 \mathrm{~Hz}$ for both trapping and ACEO flow. This implied that the main concentration mechanism was indeed ACEO flow. 


\subsubsection{Optimization of through flow velocity}

A higher through-flow will bring in more algae for trapping, which could increase the number of trapped algae and produce stronger fluorescence for algal monitoring. However, too fast flow velocity will make trapping more difficult, because the flow drag force on algae gets stronger and the time for the algae passing through the trapping electrode becomes shorter. There is, therefore, a trade-off for an optimized flow velocity of concentrating the algae. In order to maximize trapped algae per unit time, the through flow velocity was optimized as well. The optimization of through flow rate was done in two steps. First, the percentage of algae becoming trapped was found for a range of flow velocities. Then an optimal flow velocity was found to achieve maximal amount of total trapped algae in a given period of time.

In the first step, algae trapping was characterized against a range of flow velocities. To find the percentage of algae being trapped, a hemocytometer was used to measure the algae densities at the outlet of Channel 1 . When the sample was pumped through Channel 1 , sedimentation by gravity also trapped some particles in the channel. In order to factor out the sedimentation effect, control experiments were run without applying an AC signal and the particle densities at the outlet were counted. Another experiment immediately followed with AC signal applied, and the particle densities were counted again. A frequency of $500 \mathrm{~Hz}$ and $2 \mathrm{~V}$ AC signal were used for the experiment. Figure 7 gives algae concentrations at the Channel 1 outlet for various external flow velocities. For every flow rate, there are two columns showing the particle densities. The left columns are the results from control 
experiments, in which no AC signal was applied, and they account for the particles lost in the channel due to sedimentation. The control columns became lower with decreasing flow rate, indicating that more sedimentation happened from longer time to pass the channel. The right columns are results with ACEO and nDEP effects. The differences between the left and right columns represent the amounts of particles trapped via ACEO and nDEP effects into Channel 2. Then that number was divided with algae density in the control experiment to obtain the percentage of algae trapped, i.e. the trapping efficiency.

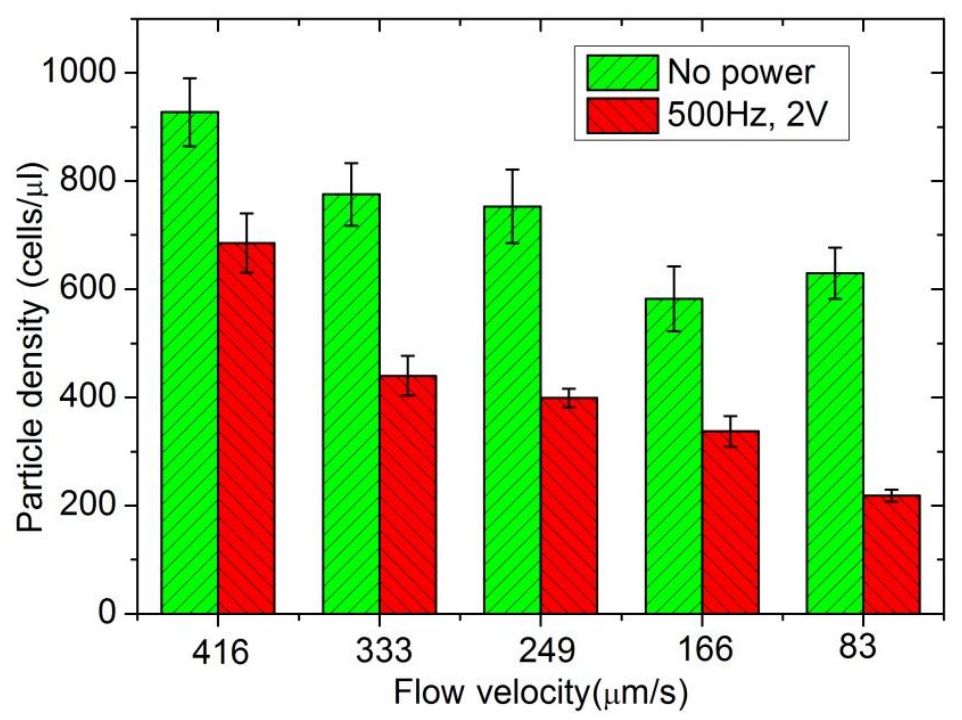

Figure 7.Algae concentrations at the Channel 1 outlet at various external flow velocities. For every flow rate, there are two columns showing the particle densities. The left columns are the results from control experiments, in which no AC signal was applied. The right columns are results from an active particle concentrator, i.e. with AC signal on. The applied AC signal is $500 \mathrm{~Hz}, 2 \mathrm{~V}$ for all experiments. The inlet particle density is fixed at 1266 cells $/ \mu \mathrm{L}$.

From Fig. 7, it can be seen that both sedimentation and ACEK trapping are more pronounced at lower flow rates, because the time for the sample transit through the channel was longer (i.e. longer time for ACEO effect and sedimentation) and more particles were 
available for trapping. At the flow velocity of $83 \mu \mathrm{m} / \mathrm{s}$, the ACEO trapping effect far exceeds sedimentation, and the sedimentation velocity of algae, estimated at $15 \mu \mathrm{m} / \mathrm{s}$, is much smaller than the ACEO velocity. ACEO flows dominate the local flow field around the mesh electrodes at low external flow velocity. At higher flow velocity, the difference in trapping between the ACEO and gravity becomes less, since external flow becomes more important. The percentage number in Table 1 represents the trapping efficiency, which was calculated as $\left(D_{n p}-D_{p}\right) / D_{n p}$, where $D_{n p}$ was the algae density at the Channel 1 outlet without power, and $D_{p}$ was the algae density at the Channel 1 outlet with the AC signal on. The highest trapping efficiency of $65.3 \%$ is obtain at a linear flow velocity of $83 \mu \mathrm{m} / \mathrm{s}$. The contribution of sedimentation has been excluded.

\section{Table 1}

Tapping efficiency vs Flow rate

\begin{tabular}{llll}
\hline Flow rate $(\mu \mathrm{l} / \mathrm{min})$ & Flow velocity $(\mu \mathrm{m} / \mathrm{s})$ & Trapping efficiency $(\%)$ & $\begin{array}{l}\text { Particle trapped per } \\
\text { unit time (cells/s) }\end{array}$ \\
\hline 25 & 416 & 26.1 & 138 \\
20 & 333 & 43.3 & 181 \\
15 & 249 & 47.1 & 149 \\
10 & 166 & 42.0 & 89 \\
5 & 83 & 65.3 & 69 \\
\hline
\end{tabular}

However, a high trapping efficiency does not necessarily provided a high amount of trapped particles, due to the fact that only a smaller amount of sample can be processed at a slow flow rate. When a high total amount of trapped particle is desired, such as in the case of capturing algae from river water, a high throughput of sample is needed. At higher flow velocity, more algae pass through the trapping device, therefore more algae become available to be trapped per unit time. Table 1 also presents the total trapped particles at various external 
flow rates. The highest concentration effect (trapped particle number per unit time) is obtained at a linear flow velocity of $333 \mu \mathrm{m} / \mathrm{s}$. With an algae concentration of 1266 cells $/ \mu \mathrm{l}$ at Channel inlet 1 , the maximum trapping rate of this device was 181.5 cells $/ \mathrm{s}$ at $333 \mu \mathrm{m} / \mathrm{s}$.

\subsubsection{Optimization of electrode mesh dimension}

In this trapping device, ACEO velocity was not only influenced by the applied frequency but by the mesh electrode dimensions. As described in Section 2, electrical field strength influences the magnitude of both ACEO and DEP effects. Particle trapping into the lower channel is the combined effect of ACEO and $\mathrm{nDEP}$, and thus is affected by the electric field, which in turn is affected by the dimensions of the mesh electrodes.

In order to find the optimal dimensions for the mesh electrode, three different opening sizes were compared for trapping effects. The sizes of opening for the three mesh electrodes were $45 \mu \mathrm{m}, 85 \mu \mathrm{m}$ and $120 \mu \mathrm{m}$, respectively. The electrode diameters were $20 \mu \mathrm{m}, 35 \mu \mathrm{m}$ and $45 \mu \mathrm{m}$, respectively. The applied frequency and voltage were $2 \mathrm{~V}$ and $500 \mathrm{~Hz}$. The external flow rate was $15 \mu \mathrm{l} / \mathrm{min}$. The results were quantified by measuring the gray scale of the microscope image shown in Fig. 8 (a). 


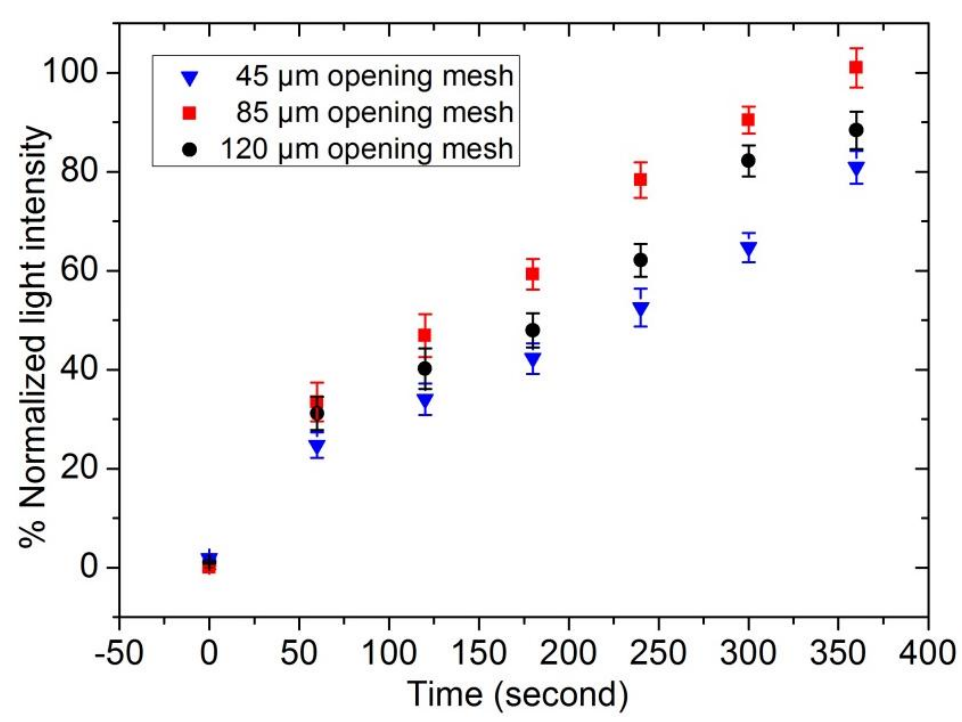

(a)

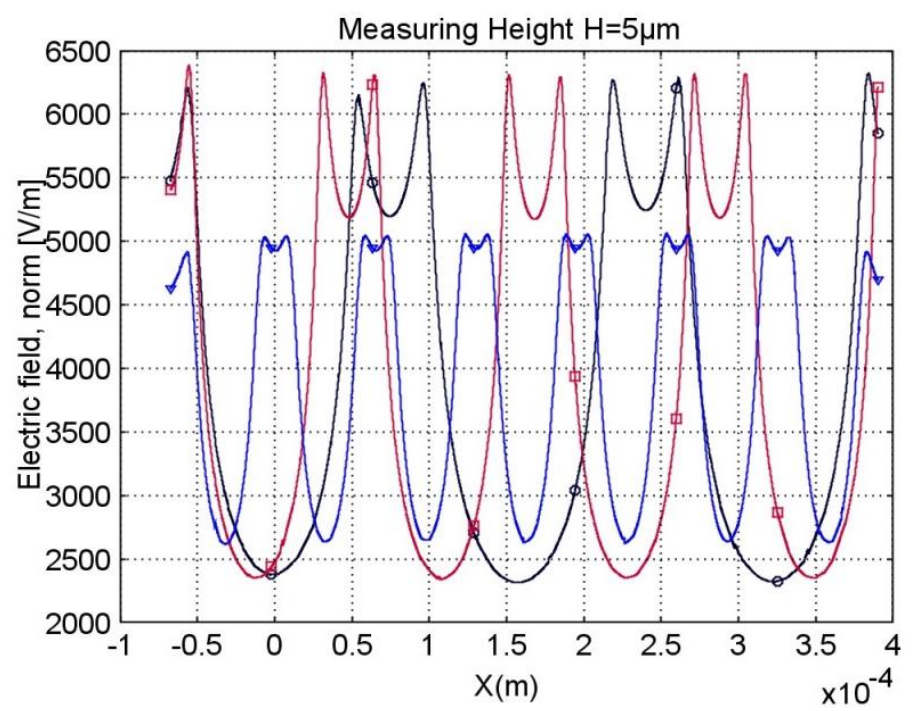

(b)

Fig. 8. (a) Experimental results of trapping with three different mesh electrodes. (b) The simulated electricfield strength in a selected cross-section area along the fluid moving direction (x-direction) at a height of $\mathrm{H}=5 \mu \mathrm{m}$ from surface of electrode. Comparisons were made between three mesh electrodes: (i) opening of $45 \mu \mathrm{m}$ with $20 \mu \mathrm{m}$ width (blue triangle), (ii) opening of $85 \mu \mathrm{m}$ with $35 \mu \mathrm{m}$ width (red square), opening of $120 \mu \mathrm{m}$ with $45 \mu \mathrm{m}$ width (black circle).

From Fig. 8 (a), it can be seen that the mesh electrode with an $85 \mu \mathrm{m}$ opening shows the best trapping effect. Compared with $45 \mu \mathrm{m}$ opening, a larger opening of $85 \mu \mathrm{m}$ leads to a larger tangential electric field as every mesh wire causes curvatures in electric field 
distribution, which will generate stronger ACEO flows. However, the electrical field curvature does not monotonically increase with the opening size. Once the opening exceeds a certain size, the number of effective openings per unit area decreases without the benefit of increasing ACEO flow, and the region affected by ACEO vortices become less. This is a tradeoff.

Experimentally, for these three sizes of mesh electrodes, the one with openings of 85 $\mu \mathrm{m}$ was found to produce the best trapping effect, as shown in Fig. 8 (a). An explanation can be found by checking how the tangential component of electrical field strength was influenced by the three electrode dimensions, which largely determines the magnitude of ACEO flows produced by parallel plate electrodes. The electrical field distribution in a plane along the central line of a mesh electrode was simulated with COMSOL Multiphysics for all three mesh electrodes. As shown in Fig. 8 (b), the simulation was performed with a 2-dimensional model covering a $470 \mu \mathrm{m}$ length along the sample injection direction (x-axis), and the $y$-axis shows the amplitude of electrical field strength tangential to the electrode surface. A periodic boundary condition was used to represent the electrical field distribution for the entire device. The tangential electrical field was measured at a height $\mathrm{H}=5 \mu \mathrm{m}$ above the electrode surface, and the results were summarized in Fig. 8 (b). It can be observed that the electrode with openings $120 \mu \mathrm{m}, 85 \mu \mathrm{m}$ presented greater electric field peak values than the $45 \mu \mathrm{m}$ mesh electrode. The mesh electrodes with $120 \mu \mathrm{m}$ and $85 \mu \mathrm{m}$ openings had similar values, however the mesh electrode with $85 \mu \mathrm{m}$ opening had more trapping sites than the 120 $\mu \mathrm{m}$ opening, which also affect the trapping effect. The simulation result shows that the 
average tangential electric field for these three mesh electrodes are $3.792 \times 10^{3} \mathrm{~V} / \mathrm{m}$, $3.840 \times 10^{3} \mathrm{~V} / \mathrm{m}$ and $3.822 \times 10^{3} \mathrm{~V} / \mathrm{m}$, respectively, agreeing with the experimental trapping results from each type mesh electrode. This further proves that the main trapping mechanism for our device is ACEO flow, and the magnitude of ACEO flow is directly related to the tangential electric field.

In addition, channel height also has effects on trapping efficiency from two aspects. Channel height will affect the flow resistance of Channel 1 presented to the pump, and also will determine the electric field strength between the electrodes. On one hand, the channel needs to be high enough to achieve reasonable through flow and avoid posing too high a flow resistance to the pump. On the other hand, only limited voltage can be applied between the electrodes without causing electrochemical reactions. Therefore, channel height cannot be so great that ACEO velocity becomes too weak for the device to function. After some tests on several channels with different heights, we found that the optimal channel height is between $300 \mu \mathrm{m}$ to $500 \mu \mathrm{m}$. A channel height of $500 \mu \mathrm{m}$ is used in this study.

\section{Conclusion}

This paper presents the development and experimental demonstration of a lab-on-a-chip based resettable particle concentration by ACEK. We have demonstrated proof of concept by successfully concentrating Chlorella algae then releasing the sample. The improvements of our device compared with conventional devices are lower limit of detection, low cost, portability and reuse. Optimization of applied frequency, external flow velocity and 
mesh opening size have been conducted to maximize the trapping effect. Table 2 represents the comparison results of our work and other peer reviewed resettable non-contact particle trapping method.

Table 2 Comparison of resettable non-contact particle trapping methods

\begin{tabular}{|c|c|c|c|c|}
\hline $\begin{array}{l}\text { Trapping } \\
\text { method }\end{array}$ & Hydrodynamic & $\begin{array}{l}\text { Insulated-based } \\
\text { Dielectrophoresis } \\
\text { (iDEP) }\end{array}$ & Acoustic & AC Electrokinetics \\
\hline $\begin{array}{l}\text { Trapping } \\
\text { efficiency }\end{array}$ & $24.3 \%-98.57 \%$ & $>20 \%$ & $>96 \%$ & $>65.3 \%$ \\
\hline $\begin{array}{l}\text { Enrichment } \\
\text { rate }\end{array}$ & 0.24 fold $/ \mathrm{min}$ & NA & $4.3 \mathrm{fold} / \mathrm{min}$ & $2 \mathrm{fold} / \mathrm{min}$ \\
\hline $\begin{array}{c}\text { System } \\
\text { complexity }\end{array}$ & $\begin{array}{l}\text { Precise flow control } \\
\text { by multiple pumps }\end{array}$ & $\begin{array}{l}\text { Require large DC } \\
\text { power supply }\end{array}$ & $\begin{array}{l}\text { High frequency } \\
\text { power supply } \\
\text { required. Sheath } \\
\text { flow required }\end{array}$ & $\begin{array}{l}\text { Low } \\
\text { voltage/frequency. } \\
\text { Can be automated. }\end{array}$ \\
\hline Portability & No & No & No & Yes \\
\hline $\begin{array}{c}\text { Target } \\
\text { particles }\end{array}$ & $\begin{array}{l}1-3 \mu \mathrm{m}, 80-200 \mu \mathrm{m} . \\
\text { Device dimensions } \\
\text { determine trapped } \\
\text { particle sizes. }\end{array}$ & Above $1 \mu \mathrm{m}$ & $\begin{array}{l}\text { Trapping sensitive } \\
\text { to particle sizes. For } \\
\text { particles larger than } \\
10 \mu \mathrm{m}\end{array}$ & Above $1 \mu \mathrm{m}$ \\
\hline Reference & {$[17][18]$} & [29-31] & {$[24][25]$} & This work \\
\hline
\end{tabular}

The experiments also showed that the trapped particles increase linearly with time, so higher power enrichment can be obtained by longer running time. In a separate experiment, the particle concentrator was run for an hour with more than 100 folds enrichment. The concentration of naturally occurring microalgae is less than $1.5 \mu \mathrm{g} / \mathrm{mL}$ in source waters [4], and microalgal autofluorescence at this concentration is approaching the limit of detection for many photodetectors. By adding 3 minutes for collection of algae, algae concentration can be brought up to $10 \mu \mathrm{g} / \mathrm{mL}$ or higher, which will make the detection much more reliable and low 
cost detectors can be used as well. Further, while the addition of several minutes for the concentration step obviously increases the minimal time interval required to obtain each fluorescence reading, this lengthening of the sampling procedure by a few minutes has a relatively small impact compared to the other factors that control response time of the algae and their photosystem II reaction centers to toxic substances. Although the ideal sampling time for water safety monitoring would be "instantaneous", the actual time intervals required are defined by (1) the total time required to obtain the fluorescence induction curves for each sample and (2) the time required for a toxin to detectably impact the algal photosynthetic machinery. Actual time used for algal detection of toxins is on the order of $30-45 \mathrm{~min}$, so an additional 3-min interval for concentration will have a minimal impact. Testing of field-deployed units in the Clinch River in Tennessee found that the minimum dark adaptation time for the natural riverine algae was $30 \mathrm{~min}$ for reversal of naturally occurring photoinhibition of microalgal autofluorescence due to incident sunlight during daylight hours. Therefore, the developed resettable particle concentrator can be easily incorporated into the protocol of monitoring microalgal autofluorescence.

\section{Acknowledgements}

This research was supported by funding from Oak Ridge National Laboratory's (ORNL) Technology Transfer and Economic Development Maturation funding program. Additional support was provided for graduate student Q. Yuan by the University of Tennessee (UTK) 
Professional Development Award. J. Wu acknowledged support from UTK Initiatives for PON/POC Nanobiosensing. Earlier research demonstrating utilization of PAM fluorimeters for detection of toxins by algal fluorescence was supported by the Defense Advanced Research Projects Agency, U. S. Department of Defense. Additional funding for complementary microelectronics research was provided by the Office of Biological and Environmental Research's Artificial Retina Program, Office of Science, U. S. Department of Energy. Oak Ridge National Laboratory is managed by UT-Battelle LLC for the U.S. Department of Energy under contract DE-AC05-00OR22725.

This manuscript has been authored by UT-Battelle, LLC under Contract No. DE-AC05-00OR22725 with the U.S. Department of Energy. The United States Government retains and the publisher, by accepting the article for publication, acknowledges that the United States Government retains a non-exclusive, paid-up, irrevocable, world-wide license to publish or reproduce the published form of this manuscript, or allow others to do so, for United States Government purposes. The Department of Energy will provide public access to these results of federally sponsored research in accordance with the DOE Public Access Plan (http://energy.gov/downloads/doe-public-access-plan). 


\section{References:}

[1] M. Rodriguez, Jr., E. Greenbaum, Detection limits for real-time source water monitoring using indigenous Freshwater microalgae, Water Environ. Res. 81 (2009) 2364-2371.

[2] C.K. Lockert, K.D. Hoagland, and B.D. Siegfried, Comparative Sensitivity of Freshwater Algae to Atrazine, Bull. Environ. Contam. Toxicol. 76 (2006) 73-79.

[3] F. Seguin, F. L. Bihan, C. Leboulanger, A. Berard, A risk assessment of pollution: induction of atrazine tolerance in phytoplankton communities in freshwater outdoor mesocosms, using chlorophyll fluorescence as an endpoint, Water Res. 36 (2002) 3227-3236.

[4] U.S. Environmental Protection Agency, Water sector security workshop, 2006

[5] U. Schreiber, U. Schliwa and W. Bilger, Continuous recording of photochemical and non-photochemical chlorophyll fluorescence quenching with a new type of modulation fluorometer, Photosynthesis Res. 10 (1986) 51-62.

[6] U. Schreiber, L. Grobermann, and W. Vidaver, Portable, solid-state fluorometer for the measurement of chlorophyll fluorescence induction in plants, Rev. Sci. Instrum. 46 (1975) 538-542.

[7] E. Ogren and N. I. Baker, Evaluation of a technique for the measurement of chlorophyll fluorescence from leaves exposed to continuous light, Plant Cell Environ. 8 (1985) 539-547.

[8] U. Schreiber, Detection of rapid induction kinetics with a new type of high-frequency modulated chlorophyll fluorometer, Photosynthesis Res. 9 (1986) 261-272.

[9] J. Cosgrove, M. Borowitzka, Applying Pulse Amplitude Modulation (PAM) fluorometry to microalgae suspensions: stirring potentially impacts fluorescence, Photosynth. Res. 88 (2006) 343-350.

[10] P. Mazzinghi, A laser diode fluorometer for field measurements of the F685/F730 chlorophyll fluorescence ratio, Rev.Sci.Instrum. 67 (1996) 3737-3744. 
[11] A. Barocs, L. Kocsany, S. Varkony, P. Richter, Z. Csintalan and K. Szente, Two-wavelength, multipurpose, truly portable chlorophyll fluorometer and its application in field monitoring of phytoremediation, Meas. Sci. Technol. 11 (2000) 717-729.

[12] A. Ashkin, J.M. Dziedzic, T. Yamane, Optical trapping and manipulation of single cells using infrared laser beams, Nature 330 (1987) 769-771.

[13] P. Jordan, J. Leach, M. Padgett, P. Blackburn, N. Isaacs, M. Goksor, D. Hanstorp, A. Wright, J. Girkin, J. Cooper, Creating permanent 3D arrangements of isolated cells using holographic optical tweezers, Lab Chip 5 (2005) 1224-1228.

[14] Y. Liu, D.K. Cheng, G.J. Sonek, M.W. Berns, C.F. Chapman, and B.J. Tromberg, Evidence for localized cell heating induced by infrared optical tweezers, Biophys.J. 68 (1995) 2137-2144.

[15] A. Khademhosseini, J. Yeh, G. Eng, J. Karp, H. Kaji, J. Borenstein, O.C. Farokhzad, R. Langer, Cell docking inside microwells within reversibly sealed microfluidic channels for fabricating multiphenotype cell arrays, Lab Chip 5 (2005) 1380-1386.

[16] H. Chen, B. Sun, K. K. Tran, H. Shen, D. Gao, A Microfluidic Manipulator for Enrichment and Alignment of Moving Cells and Particles, J. Biomech. Eng. 131 (2009) 074505-1.

[17] C. Kim, J. H. Bang, Y. E. Kim, J. H. Lee, J. Y. Kang, Stable hydrodynamic trapping of hydrogel beads for on-chip differentiation analysis of encapsulated stem cells, Sens. Actuators B 166 (2012) 859-869.

[18] M. Yamada, M. Seki, Hydrodynamic filtration for on-chip particle concentration and classification utilizing microfluidics, Lab Chip 5 (2005) 1233-1239.

[19] T. Kimura, Y. Sato, F. Kimura, M. Iwasaka, S.Ueno, Micropatterning of cells using modulated magnetic fields, Langmuir 21 (2005) 830-832.

[20] Martin A.M. Gijs, Magnetic bead handling on-chip: new opportunities for analytical applications, J. Microfluid. Nanofluid 1 (2004) 22-40. 
[21] T. Lund-Olesen, M. Dufva, M.F. Hansen, Capture of DNA in microfluidic channel using magnetic beads: Increasing capture effieciency with integrated microfluidic mixer, J.Magn.Magn. Mater. 311 (2007) 396-400.

[22] H. Lee, A.M. Purdon, R.M. Westervelt, Manipulation of biological cells using a microelectromagnet matrix, Appl. Phys. Lett.85 (2004) 1063-1065.

[23] A. R. Kose, B. Fischer, L. Mao, and H. Koser, Label-free cellular manipulation and sorting via biocompatible ferrofluids, PNAS, 106 (2009) 21478-21483.

[24] M. Evander, L. Johansson, T. Lilliehorn, J. Piskur, M. Lindvall, S. Johansson, M. Almqvist, T. Laurell, J. Nilsson, Noninvasive acoustic cell trapping in a microfluidic perfusion system for online bioassays, Anal. Chem. 79 (2007) 2984-2991.

[25] B. Hammarstrom, M. Evander, H. Barbeau, M. Bruzelius, J. Larsson, T. Laurell, J.Nilsson, Non-contact acoustic cell trapping in disposable glass capillaries, Lab Chip 10 (2010) 2251-2257.

[26] D. Holmes, N.G. Green, H. Morgan, Microdevices for dielectrophoretic flow-through cell separation, IEE. Eng. Med. Biol. Mag. 22 (2003) 85-90.

[27] H. Shafiee, J. L. Caldwell, M.B. Sano, R.V. Davalos, Contactless dielectrophoresis: a new technique for cell manipulation, Biomed.Microdevices. 11 (2009) 997-1006.

[28] S. Li, Q. Yuan, C. Ke, J. Wu, Dielectrophoretic Response of DNA and Fluorophore in Physiological Solution by Impedimetric Characterization, Biosens. Bioelectron. 41 (2013) 649-655.

[29] E.B. Cummings, A.K. Singh, Dielectrophoresis in microchips containing arrays of insulating posts: theoretical and experimental results, Anal.Chem.75 (2003) 4724-4731.

[30] B.H. Lapizco-Encinas, B.A. Simmons, E.B. Cumming, Y. Fintschenko, Dielectrophoretic concentration and separation of live and dead bacteria in an array of insulators , Anal.Chem.76(2004)1571-1579.

[31] S. Bhattacharya, T. Chao, N. Ariyasinghe, Y. Ruiz, D. Lake, R. Ros, A. Ros, Selective trapping of single mammalian breast cancer cells by insulator-based dielectrophoresis, Anal. Bioanal. Chem. 406 (2014) 1855-1865. 
[32] J.Wu, Interactions of Electrical Fields with Fluids: Laboratory-on-a-chip Applications, IET Nanobiotechnol. 2 (2008) 14-27.

[33] M.Z.Bazant, AC Electro-osmotic flow, Encyclopedia of Microfluidics and Nanofluidics., New York, 2008, pp. 8-14.

[34] J.Wu,N. Islam, M. Lian, High sensitivity particle detection by biased AC electro-osmotic trapping on cantilever, $19^{\text {th }}$ IEEE Int. Conf. Micro. Electro. Mech.Sys. MEMS, 22-26 (2006) 566-569.

[35] A. Ramos, H. Morgan, N.G. Green, A. Castellanos, AC electric-field-induced fluid flow in microelectrodes, J. Colloid interface Sci. 217 (1999) 420-422.

[36] J. Wu, Y. Ben, H.C. Chang, Particle detection by electrical impedance spectroscopy with asymmetric polarization AC electroosmotic trapping, J. Microfluid. Nanofluid. 1 (2005) 161-167.

[37] J. Wu, Y. Ben, D. Battigelli, H.C. Chang, Long-range AC electroosmotic trapping and detection of bioparticles, Ind. Eng. Chem. Fundam. 44 (2005) 2815-2822.

[38] J. Wu and N. Islam, A simple method to integrate in-situ nano-particle focusing with cantilever detection, IEEE Sens. J. 7 (2007) 957-958.

[39] N. Islam, M. Lian, and J. Wu, Enhancing cantilever capability with integrated AC electrokinetic trapping mechanism, J. Microfluidics \& Nanofluidics 3 (2007) 369-375.

[40] V. Studer, A. Pépin, Y. Chen, and A. Ajdari, An integrated AC electrokinetic pump in a microfluidic loop for fast and tunable flow control, Analyst. 129 (2004) 944-949.

[41] S. Li, H. Cui, Q. Yuan, J. Wu, A. Wadhwa, S. Eda, AC electrokinetics-enhanced capacitive immunosensor for point-of-care serodiagnosis of infectious diseases, Biosens. Bioelectron. 51 (2014) 437-443.

[42]N. Sasaki, T. Kitamori, HB.Kim, AC electroosmotic micromixer for chemical processing in a microchannel, Lab chip 6 (2006) 550-554.

[43]S. Huang, S. Wang, H. Khoo, F. Tseng, AC electroosmotic generated in-plane microvortices for stationary or continuous fluid mixing, Sens. Actuators B 125 (2007) 326-336. 
[44] W.Y. Ng, S. Goh, Y. Lam, C. Yang, I. Rodriguez, DC-biased AC-electroosmotic and AC-electrothermal flow mixing in microchannels, Lab chip 9(2009) 802-809.

[45] J. Chen, C. Weng and R. Yang, Assessment of three AC electroosmotic flow protocols for mixing in microfluidic channel, Lab chip 9 (2009) 1267-1273.

[46] M. Lian, N. Islam, J. Wu, AC electrothermal manipulation of conductive fluids and particles for Lab-chip applications, IET Nanobiotechnology 1 (2007) 36-43.

[47] Q. Yuan, K. Yang, J. Wu, Optimization of planar interdigitated microelectrode array for biofluid transport by AC electrothermal effect, J. Microfluidics \& Nanofluidics 16 (2014) 167-178.

[48]K. Yang, and J. Wu, Investigation of microflow reversal by ac electrokinetics in orthogonal electrodes for micropump design, Biomicrofluidics 2 (2008) 024101

[49]N. Sueoka, Mitotic replication of deoxyribonucleic acid in chlamydomonas reinhardi, Proc. Natl. Acad. Sci. 46 (1960) 83-91.

[50] R.J. Porra, Spectrometric assays for plant, algal and bacterial chlorophylls, Advances in Photosynthesis and Respiration 25 (2006) 95-107.

[51]C. Cheng, S. Wang, J. Wu, Y. Yu, R. Li, S. Eda, J. Chen, G. Feng, B. Lawrie and A. $\mathrm{Hu}$, Bisphenol A Sensors on Polyimide Fabricated by Laser Direct Writing for Onsite River Water Monitoring at Attomolar Concentration, ACS Appl.Mater.Inter. 8 (2016) 17784-17792 